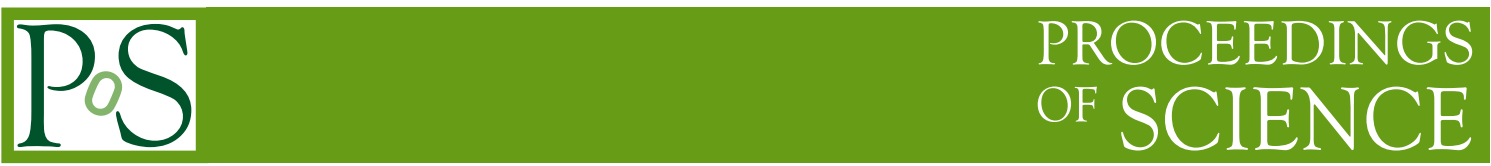

\title{
Gamma-ray emission from AP Librae (PKS1514-241)
}

\section{Pascal Fortin ${ }^{a}$, Stephen Fegan ${ }^{a}$, Deirdre Horan ${ }^{a}$, David Sanchez ${ }^{a, e}$, on behalf of the Fermi-LAT Collaboration \\ Berrie Giebels $^{a}$, Yvonne Becherini ${ }^{a, b}$, Guillaume Dubus ${ }^{c}$, Mathieu de Naurois ${ }^{a}$, Michael Punch ${ }^{b}$, Martin Raue ${ }^{d}$, on behalf of the H.E.S.S. Collaboration}

${ }^{a}$ Laboratoire Leprince-Ringuet, École Polytechnique, CNRS / IN2P3, Palaiseau, France

${ }^{b}$ Astroparticule et Cosmologie, CNRS, Université Paris 7 Denis Diderot, Paris Cedex 13, France

${ }^{c}$ UJF-Grenoble 1 / CNRS-INSU, Institut de Planétologie et d'Astrophysique de Grenoble,

Grenoble, France

${ }^{d}$ Universität Hamburg, Institut für Experimentalphysik, Hamburg, Germany

${ }^{e}$ Max-Planck-Institut für Kernphysik, Heidelberg, Germany

E-mail: fortinallr.in2p3.fr

The BL Lac type object AP Librae $(z=0.049)$ was detected at the 7 standard deviation level in $11 \mathrm{~h}$ of live time observations with the High Energy Stereoscopic System (H.E.S.S.) in the summer of 2010. The Fermi-LAT source 1FGL J1517.8-2423, associated with AP Librae, has a hard $\gamma$ ray spectrum with a photon index of $2.1 \pm 0.1$. No flux variability is detected in the H.E.S.S. data set nor in 25 months of Fermi-LAT data. From the broad-band spectral-energy distribution AP Librae has been classified as a low-frequency-peaked BL Lac. Archival and contemporaneous Xray observations by the Swift and RXTE satellites show a hard energy spectrum which, together with the flat Fermi-LAT spectrum and the H.E.S.S. detection at very-high energies (VHE, $E \geq$ $100 \mathrm{GeV}$ ), indicate an unusually broad high-energy component stretching from X-rays to VHE $\gamma$-rays, wider than all other VHE $\gamma$-ray emitting LBL, making AP Librae a unique VHE emitter.

25th Texas Symposium on Relativistic Astrophysics - TEXAS 2010

December 06-10, 2010

Heidelberg, Germany 


\section{Introduction}

The vast majority of very-high-energy (VHE, $E \geq 100 \mathrm{GeV}$ ) $\gamma$-ray-emitting active galaxies belong to the BL Lac class ${ }^{1}$, itself subdivided into high-frequency, intermediate-frequency and low-frequency peaked BL Lacs (HBL, IBL, and LBL respectively) depending on the peak position of the $v F_{v}$ synchrotron component in the spectral energy distribution (SED): in UV/X-rays for HBL, in the IR/UV range for IBL, and in the IR or lower for LBL. The VHE $\gamma$-ray emitters are mostly HBL, with AP Librae being only the third LBL seen by an atmospheric Cherenkov telescope (ACT) after BL Lacertae [1] and S5 0716+714 [2]. LBLs tend to have higher luminosities, are mostly found in radio surveys (while HBLs are mostly found in X-ray surveys), and might be a distinct class from HBLs though there could be a continuity between the LBL and HBL as suggested by the "blazar sequence" where the dominance of the high-energy component and its peak emission energy is anticorrelated with the total luminosity [8]. The multiwavelength characterization of these blazars is of paramount importance for the development of models which explain the broad band emission, from millimeter to VHE energies, as originating from a dissipative region in a highly relativistic jet.

\section{Observations and data analysis}

AP Librae was targeted for H.E.S.S. observations after it was reported in the Fermi-LAT Bright Source List (0FGL J1517.9-2423) [7] with a hard photon index $(\Gamma \sim 2)$. The extrapolation of its spectrum into the VHE energy range predicted detectable levels of VHE $\gamma$-rays. The FermiLAT index is much harder than the index of the possible EGRET counterpart (3EG J1517-2538; $\Gamma=2.66 \pm 0.43$ ) in the third EGRET Catalog [5], which extrapolated much less favourably into the VHE $\gamma$-ray energy range, and was hence not as outstanding a VHE candidate as, e.g., PKS 2155304. Blazars can be extremely variable objects, and the source might have been softer during the EGRET exposures. Indeed, the non-detection of AP Librae by the University of Durham Mark $6 \gamma$ ray telescopes could indicate such variability [9]. It is, however, unlikely that the Mark 6 telescopes would have been sensitive enough to detect AP Librae at its current flux level as measured by H.E.S.S., which corresponds to $\sim 2 \%$ of the steady flux from the Crab nebula. However, had an ACT detected AP Librae in the EGRET period, then the spectral mismatch would doubtless have triggered intense speculations about either the understanding of the extragalactic background light (EBL) level, or of the need for multiple emission components in the inverse-Compton (IC) emission.

AP Librae was observed by H.E.S.S. from May 11, 2010 to July 10, 2010. After the initial H.E.S.S. detection at the $7 \sigma$ level in 11 hours of live time exposure [6], the X-ray component was probed jointly by the Swift/XRT and RXTE/PCA detectors. The Swift/XRT observation was performed on July 7, 2010 for a total of $\sim 5.4$ ks, while the UVOT data were taken in February and July 2010. The RXTE observations were performed between July 10, 2010 and July 14, 2010. The joint $\mathrm{X}$-ray spectrum from $0.3 \mathrm{keV}$ to $\sim 12 \mathrm{keV}$ is well represented by a power law with a good fit chi-square probability of $P\left(\chi^{2}\right)=0.98$, a hard photon index of $\Gamma=1.58 \pm 0.06$ and a $2-10 \mathrm{keV}$ flux of $F_{2-10 \mathrm{keV}}=4.9_{-0.7}^{+0.8} \mathrm{erg} \mathrm{cm}^{-2} \mathrm{~s}^{-1}$. This clearly indicates that the second component of the

\footnotetext{
${ }^{1}$ see, e.g., the TeVCat catalogue http://tevcat.uchicago.edu
} 
spectral energy distribution, usually attributed to inverse-Compton radiation, is present at energies as low as $0.3 \mathrm{keV}$. The UVOT data, taken contemporaneously with the XRT data, lie on the falling edge of the synchrotron component of the SED. The H.E.S.S. spectrum is well described by a power law of index $\Gamma_{V H E}=2.5 \pm 0.2$, compatible with an extrapolated Fermi-LAT spectrum attenuated by a Franceschini-type [3] model of the EBL.

The Fermi-LAT data for this analysis were taken from 4 August 2008 to 4 September 2010 (25 months). These data were analyzed using the standard Fermi analysis software (ScienceTools v9r19p0; IRF P6_V3_DIFFUSE) available from the Fermi Science Support Center (FSSC) ${ }^{2}$. The contribution from all 1FGL point sources within a $10^{\circ} \mathrm{ROI}$, a diffuse galactic background, and an isotropic background were modeled in the analysis. Events were analyzed using the unbinned maximum likelihood method as implemented in gtlike. AP Librae is located well within the Fermi 95\% confidence contour (see Figure 1). Remarkably, no evidence for variability in the source flux or spectral index was found during the course of these observations. The light curve is shown in Figure 2, and is entirely compatible with a constant as a function of time. While the lack of significant variability in the Fermi-LAT energy band seems increasingly to be a characteristic of HBL, this is more surprising for a LBL where the observed $\gamma$-ray Fermi-LAT variation amplitudes are usually large [10].

The energy spectrum is well described by a power law with spectral index of $\Gamma=2.1 \pm 0.1$. No evidence for spectral curvature is found. The integral flux, $F$, between $300 \mathrm{MeV}$ and $300 \mathrm{GeV}$ is $F_{0.3-300 \mathrm{GeV}}=(1.9 \pm 0.1) \times 10^{-8} \mathrm{~cm}^{-2} \mathrm{~s}^{-1}$. The most energetic photon in the Fermi-LAT data is at $160 \mathrm{GeV}$.

\section{Discussion}

One of the most striking features of the SED, compared to other VHE $\gamma$-ray emitting BL Lacs, is that the width of the high-energy component is extremely large, spanning over 33 octaves in energy, extending from $\simeq 0.1 \mathrm{keV}$ up to $\sim \mathrm{TeV}$ energies (see Figure 3 ). The synchrotron cutoff in AP Librae occurs at energies $\leq 0.1 \mathrm{keV}$, which is quite remarkable compared to the above-mentioned VHE emitting LBLs, since the Swift/RXTE X-ray band is clearly still synchrotron radiation for both BL Lacertae [11] and S5 0716+714 [2], while HBLs usually have high-energy cutoffs $>10 \mathrm{keV}$. Combined with the fact that the VHE $\gamma$-ray spectra of these other LBLs are also much steeper, the inverse-Compton portion of AP Librae's SED is probably the broadest seen in any TeV BL Lac.

Following the prescription of [4], each component of the SED has been fit with a 3rd degree polynomial in $v F_{v}$. The synchrotron and inverse-Compton peak energies are found at $E_{s}^{\text {peak }} \simeq$ $0.1 \mathrm{eV}$ and $E_{I C}^{\text {peak }} \simeq 10 \mathrm{MeV}$ respectively (as is the case for the two other above-mentioned LBLs). While the value of $E_{S}^{\text {peak }}$ is similar to that found in [4], the IC peak is well below the $E_{I C}^{\text {peak }} \simeq 10^{9} \mathrm{eV}$ which the authors predicted based on a $\Gamma_{H E}-E_{I C}^{\text {peak }}$ correlation. This is likely to come from the additional VHE data set, which was not available in that previous study. Note also that the $v F_{v}$ width of the inverse-Compton peak is nearly twice that of the synchrotron peak when compared at flux levels of $\sim 10 \%$ of the peak emission. This could be a strong indication that scattering occurs essentially in the Thomson regime, since comptonized photons then have their energy boosted by a

\footnotetext{
${ }^{2} \mathrm{http} / / /$ fermi.gsfc.nasa.gov/ssc/
} 

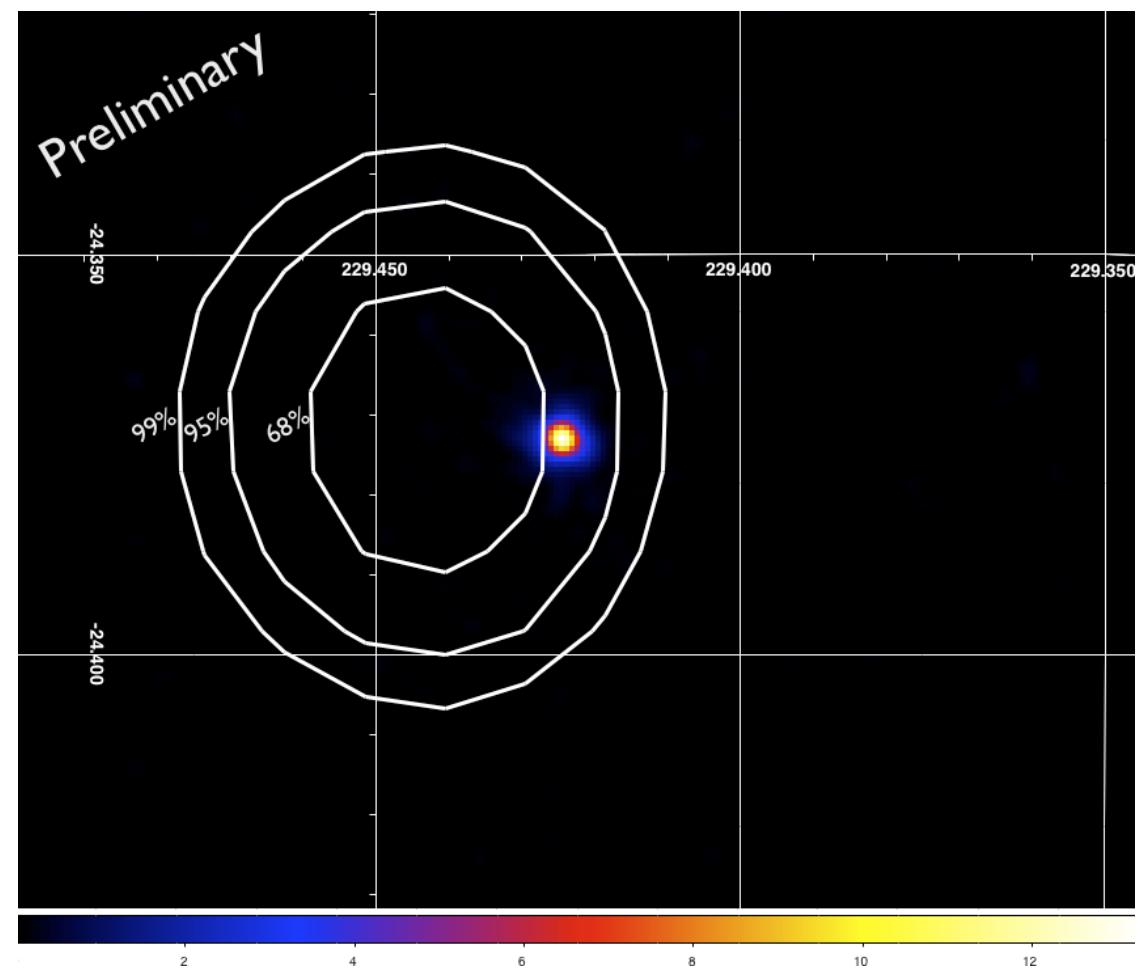

10

Figure 1: Swift/XRT count map of the region located near AP Librae. The coordinates of the centroid of the X-ray emission are consistent with the VLBI position of AP Librae. The contour lines correspond to the $68 \%-95 \%-99 \%$ confidence levels on the Fermi-LAT source localization.

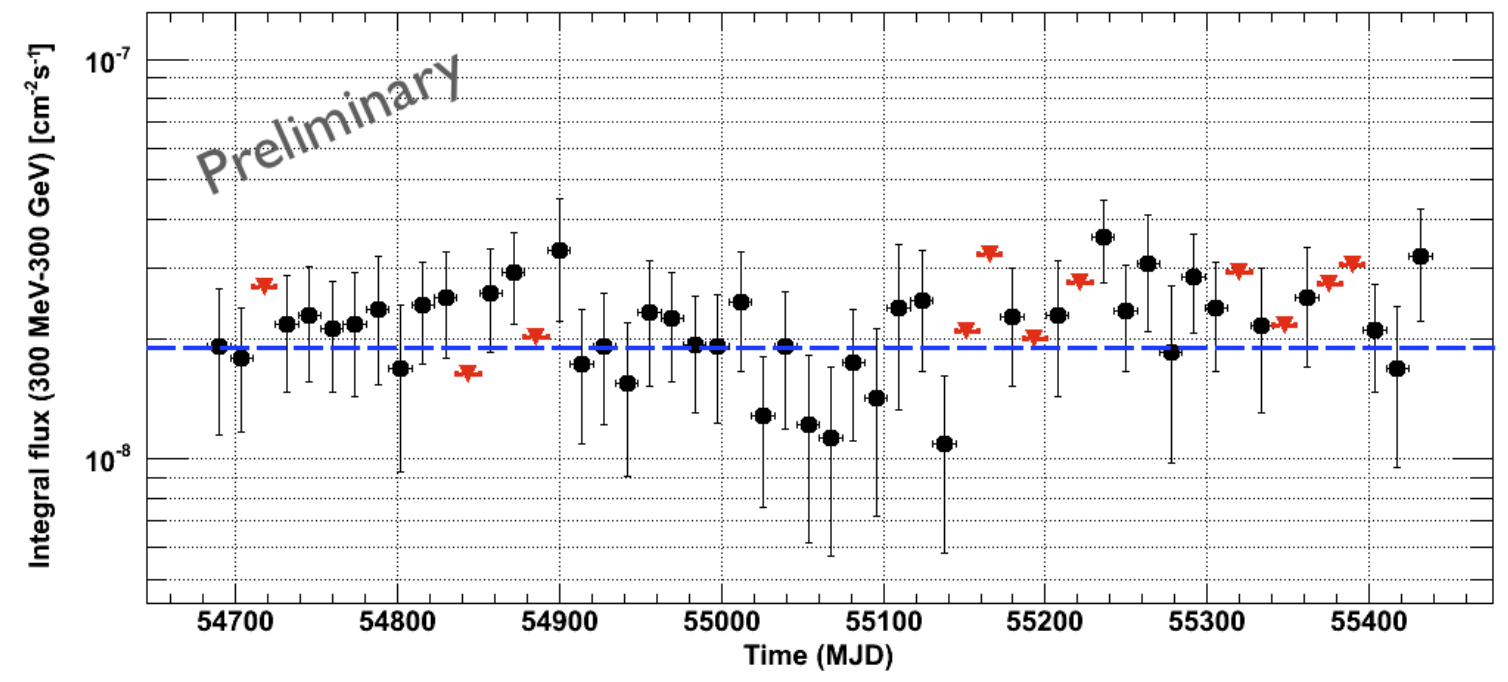

Figure 2: Fermi light curve for 25 months of data on AP Librae. No evidence for variability was found in the 14 day-binned light curve. The dashed line corresponds to the average flux for 25 months of data, while the red triangles correspond to $95 \%$ confidence level upper limits. 


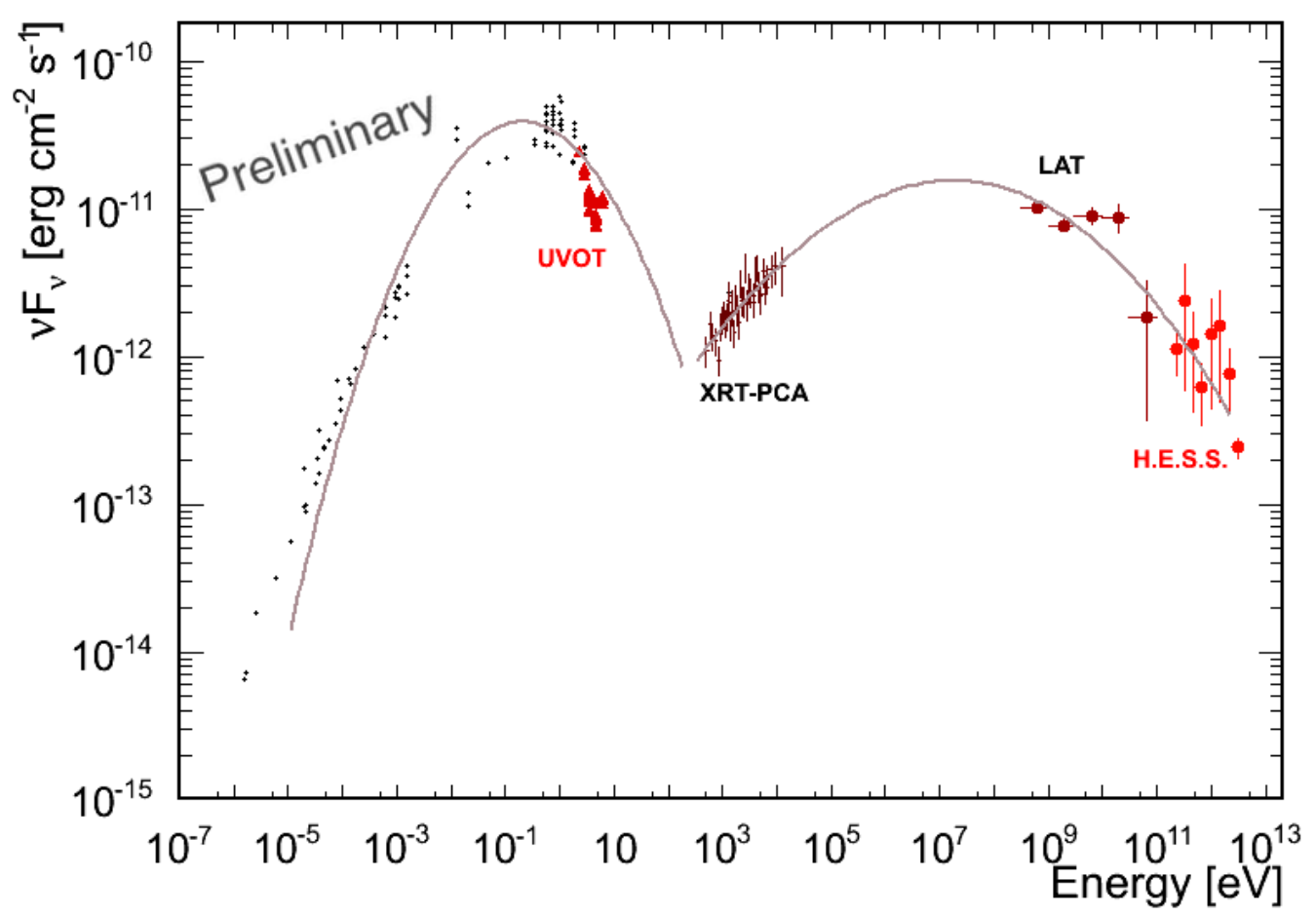

Figure 3: SED of AP Librae. The small black points below the UVOT range are archival data from NED, while the data points above the UVOT range are from contemporaneous Swift/XRT and RXTE/PCA, FermiLAT, and H.E.S.S.observations. Each component of the SED was fitted with with a 3rd degree polynomial in $v F_{v}$.

factor $\propto \gamma^{2}$, or doubled in a logarithmic scale. Such wide distributions pose serious challenges for synchrotron self-Compton type models; before the detection of any VHE signal from AP Librae and based only on the inclusion of Fermi-LAT and Swift data, Tavecchio et al. [12] experienced difficulties in reproducing the high-energy component for AP Librae and S5 0716+714. Similar difficulties arose in the modelling of LBLs once VHE $\gamma$-ray emission was included, when either multiple emission zones $[2,11]$ or an additional external photon target field [11] were necessary to account for the whole SED. Clearly, VHE $\gamma$-ray emitting LBL objects are unique objects which set new constraints on radiative emission models.

\section{Acknowledgments}

The Fermi LAT Collaboration acknowledges support from a number of agencies and institutes for both development and the operation of the LAT as well as scientific data analysis. These include NASA and DOE in the United States, CEA/Irfu and IN2P3/CNRS in France, ASI and INFN in Italy, MEXT, KEK, and JAXA in Japan, and the K. A. Wallenberg Foundation, the Swedish Research Council and the National Space Board in Sweden. Additional support from INAF in Italy and CNES in France for science analysis during the operations phase is also gratefully acknowledged. 
The support of the Namibian authorities and of the University of Namibia in facilitating the construction and operation of H.E.S.S. is gratefully acknowledged, as is the support by the German Ministry for Education and Research (BMBF), the Max Planck Society, the French Ministry for Research, the CNRS-IN2P3 and the Astroparticle Interdisciplinary Programme of the CNRS, the U.K. Science and Technology Facilities Council (STFC), the IPNP of the Charles University, the Polish Ministry of Science and Higher Education, the South African Department of Science and Technology and National Research Foundation, and by the University of Namibia. We appreciate the excellent work of the technical support staff in Berlin, Durham, Hamburg, Heidelberg, Palaiseau, Paris, Saclay, and in Namibia in the construction and operation of the equipment.

\section{References}

[1] J. Albert, et al., Discovery of VHE $\gamma$-ray emission from BL Lac, ApJ 666 (2007) L17 [astro-ph/0703084]

[2] H. Anderhub, et al., Discovery of very high energy $\gamma$-rays from S5 0716+714, ApJ 704 (2009) L129 [astro-ph/0907.2386]

[3] A. Franceschini, et al., Background radiations and the cosmic photon-photon opacity, A\&A 487 (2008) 837 [astro-ph/0805.1841]

[4] A.A. Abdo, et al., The SED of Fermi bright blazars, ApJ 716 (2010) 30 [astro-ph / 0912 . 20 040 ]

[5] R.C. Hartman, et al., The third EGRET catalog of high-energy gamma-ray sources, ApJS 123 (1999) 79

[6] W. Hofmann, The Astronomer's Telegram \#2743 (2010)

[7] A.A. Abdo, et al., Fermi/LAT bright source list, ApJS 183 (2009) 46 [a st ro-ph/ 0902 . 1340]

[8] G. Fossati, et al., A unifying view of the spectral energy distributions of blazars, MNRAS 299 (1998) 433 [astro-ph/9804103]

[9] P.M. Chadwick, et al., Search for VHE gamma rays from southern AGNs, ApJ 521 (1999) 547

[10] A.A. Abdo, et al., Gamma-ray light curves and variability of bright Fermi-detected blazars, ApJ 722 (2010) 520 [astro-ph/ 1004.0348 ]

[11] A.A. Abdo, et al., The first Fermi multifrequency campaign on BL Lac, In press ApJ (2011) [astro-ph/1101.5905]

[12] F. Tavecchio, et al., TeV BL Lac objects at the dawn of the Fermi era, MNRAS 401 (2010) 1570 [astro-ph/0909.0651] 